\title{
An Unusual Life-Threatening Hemobilia Caused by Hepatic Pseudoaneurysm Following Choledochostomy: A Case Report
}

\author{
Zijun Liu, Shiyong Yang, Pengcheng Xi \\ Nanjing First Hospital Affiliated to Nanjing Medical University, Nanjing, China \\ Email: liuzijundoctor@sina.com
}

Received 19 January 2016; accepted 15 February 2016; published 18 February 2016

Copyright (C) 2016 by authors and Scientific Research Publishing Inc.

This work is licensed under the Creative Commons Attribution International License (CC BY).

http://creativecommons.org/licenses/by/4.0/

(c) (7) Open Access

\begin{abstract}
The present report describes a case of hemobilia caused by hepatic pseudoaneurysm. A 63-yearold woman was admitted with abdominal pain and mild jaundice. She was diagnosed as choledocholithiasis and hypersplenism and underwent choledocolithotomy and splenectomy. 9th day post operation, massive fresh blood suddenly flew out from $T$ tube and she underwent emergency abdominal exploration but there were no obvious bleeding sites in the abdominal cavity and no bleeding sites in the biliary tree by choledochoscope. 7th day after the second operation, fresh blood suddenly flew out from $T$ tube again and angiography showed two small peudoaneurysms at the second branch of right hepatic artery which might result in hemobilia. The hemobilia was treated successfully with coil embolisation of peudoaneurysms and she recovers fully.
\end{abstract}

\section{Keywords}

Hemobilia, Hepatic Pseudoaneurysm, Choledochostomy

\section{Introduction}

Hemobilia is a rare, life threatening biliary tract complication. It can be caused by hepatic pseudoaneurysm [1] [2] due to both iatrogenic and noniatrogenic causes [3]. The mechanism for the formation of hepatic pseudoaneurysm is still unclear. There are several ways to treat hepatic pseudoaneurysm-induced hemoblia. However, no single treatment modality has been considered as the best treatment option for all patients [4]. Here, we report a case of hemobilia caused by hepatic pseudoaneurysm treated successfully by Transcatheter Arterial Embolization (TAE). 


\section{Case Presentation}

A 63-year-old woman was admitted to our hospital because of right upper quadrant abdominal pain radiating to back for more than one month. She had history of subtotal gastrectomy 32 years ago and open cholecystectomy and choledocholithotomy 2 years ago. She also had a 7-year history of hypertension and coronary heart disease, but had no history of hepatitis. After examination, she was found to have mild yellowish conjunctivae; body temperature $36.6^{\circ} \mathrm{C}$, blood pressure $138 / 70 \mathrm{mmHg}$, pulse rate $76 / \mathrm{min}$ and respiratory rate 16/min. Right upper quadrant tenderness was mild with no muscle rigidity. Laboratory results were as follows: white cell count 1.23 $\times 10^{9} / \mathrm{L}$ (normal, $3.5-9.5 \times 10^{9} / \mathrm{L}$ ), platelet count $36 \times 10^{9} / \mathrm{L}$ (normal, $125-350 \times 10^{9} / \mathrm{L}$ ), total bilirubin 72 umol/L (normal, 5.1 - $28 \mathrm{umol} / \mathrm{L}$ ), direct bilirubin $49.5 \mathrm{umol} / \mathrm{L}$ (normal, 0 - $10 \mathrm{umol} / \mathrm{L}$ ), alanine aminotransferase $191 \mathrm{IU} / \mathrm{L}$ (normal, 7 - 40 IU/L), aspartate aminotransferase $203 \mathrm{IU} / \mathrm{L}$ (normal 13 - 35 IU/L). Computed tomography (CT) showed dilated common bile duct (CBD) with stones inside (Figure 1), esophagogastric varicose veins, and splenomegaly (Figure 2). MRI also revealed dilated common bile duct with stones and splenomegaly (Figure 3). She was diagnosed as choledocholithiasis and hypersplenism (due to Portal Hypertension) for which underwent both choledocolithotomy and splenectomy. The CBD was explored and stones were removed. A T-tube choledochostomy was created and a drainage tube was put in the Morison's pouch.

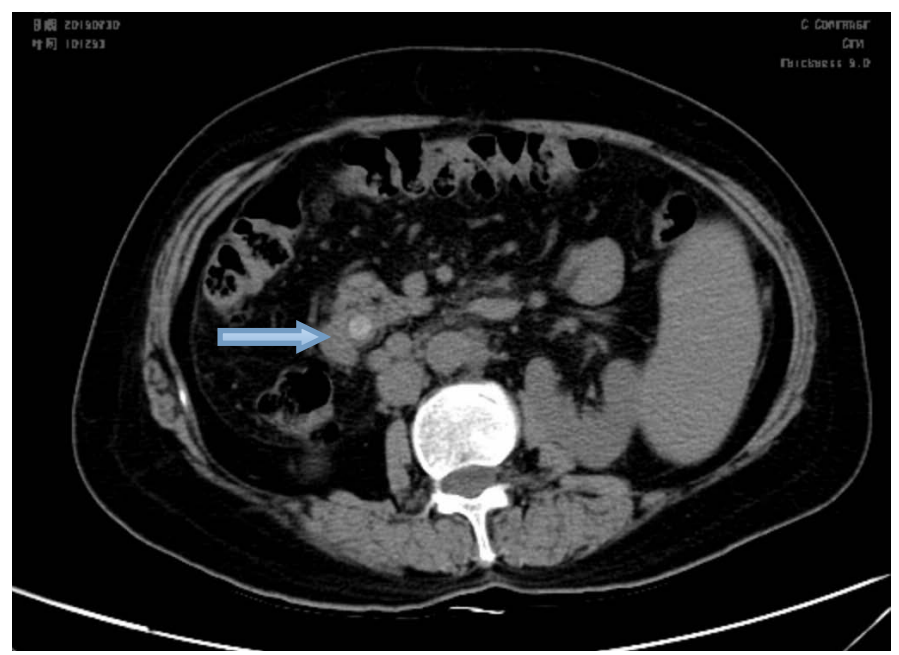

Figure 1. CT showed dilated common bile duct with stone (arrow).

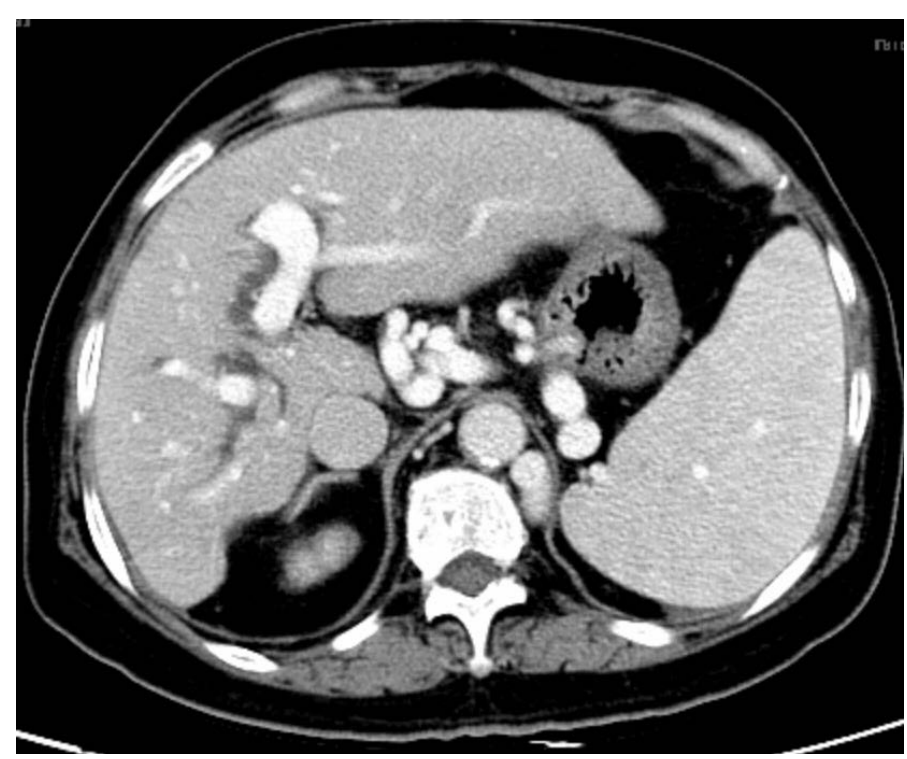

Figure 2. CT showed, esophagogastric vericose, and splenomegaly. 
The patient recovered well after the operation with normalization of both white cell and platelet counts. She had neither fever nor jaundice and was planned to leave the hospital. However, on the 9th post operative day, she developed sudden bleeding and $400 \mathrm{ml}$ fresh blood flew out from the T-tube in less than ten minutes. The T-tube was immediately clamped and three hours later she felt right abdominal pain with distention. A few amount of fresh blood flew out alongside the T-tube from the abdominal cavity. Because of high suspicion of active bleeding, she underwent emergent abdominal exploration. During operation, a large amount of blood clot at the Morison's Pouch was removed and suspicious bleeding sites were sutured. The T-tube was pulled out from CBD and fiber choledochoscopy was performed. Revealing no active bleeding or any abnormality in the whole biliary tract A new T-tube was placed in the CBD again. She recovered well. However, on the 7th day after the second operation, massive fresh blood flew out from the T-tube again. We promptly clamped the T-tube and sent the patient to the department of interventional radiology. Angiography showed two small aneurysms (2 $\times 2 \mathrm{~mm}$ and $1 \times 1 \mathrm{~mm}$ in size) at the second branch of right hepatic artery (Figure 4). On the assumption of those two attacks of hemobilia caused by the detected pseudoaneurysms; Transcatheter Embolization of the right hepatic artery was done by multiple coils at both the proximal and the distal sides of the two aneurysms (Figure 5). After TAE, she recovered uneventfully and was discharged on the 14th day post second operation. One month

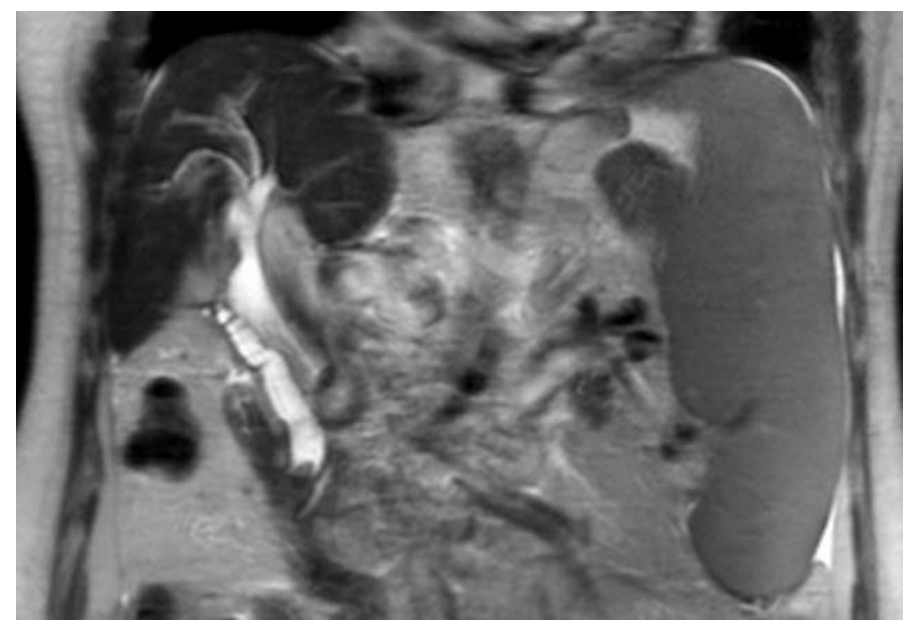

Figure 3. MRI revealed dilated common bile duct with stones and splenomegaly.

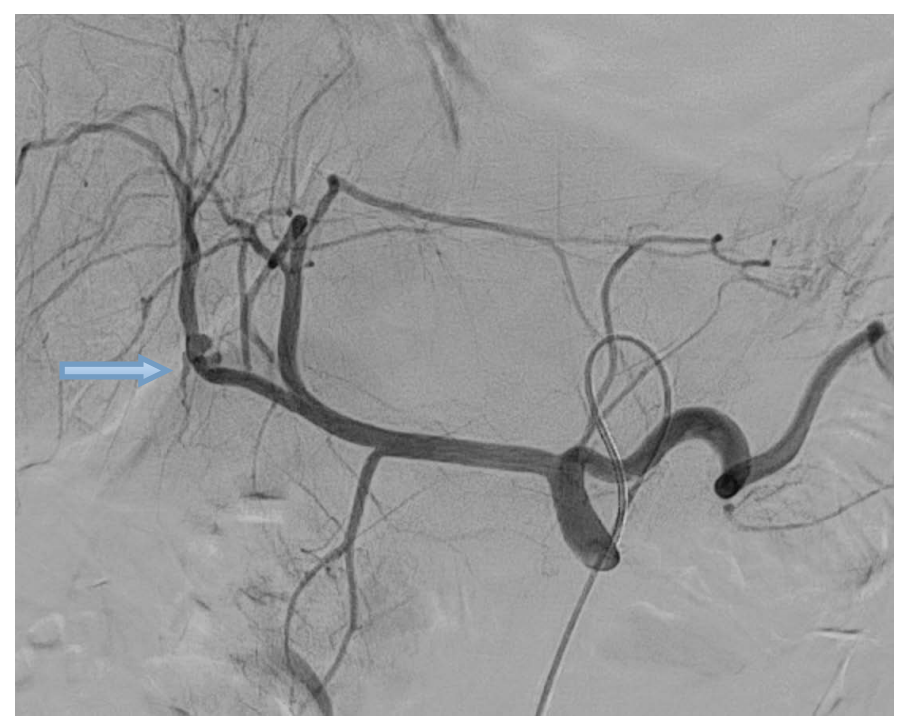

Figure 4. Two small pseudoaneurysms $(2 \times 2 \mathrm{~mm}$ and $1 \times 1 \mathrm{~mm}$ in size) of the right hepatic artery (arrow). 
after the TAE a CT examination showed multiple coils at the psedoaneurysm sites (Figure 6) with no hepatobiliary necrosis, abscess formation or biloma. Her liver enzymes profile was found normal after a three-month period of follow-up.

On reviewing the case retrospectively no pseudoaneurysm was present on the CT images prior to the first biliary surgery two years ago (Figure 7), but surprisingly the CT images available before the second biliary operation have shown two small neglected pseudoaneurysms (Figure 8).

\section{Discussion}

Hemobilia is a rare, life threatening emergency. It can be caused by many factors, including abdominal trauma, hepatobiliary surgery, liver abscess, liver biopsy, and choledocholithiasis [1]. Hepatic pseudoaneurysm is also responsible for hemobilia [2] [5]. While the mechanism for the formation of hepatic pseudoaneurysm is unclear, it has been reported that it may result from thermal or mechanical injury to the vessels during the operation [6], infiltration of the severe inflammatory reaction in the bile duct [1] or mechanical compression of the T-tube [7].

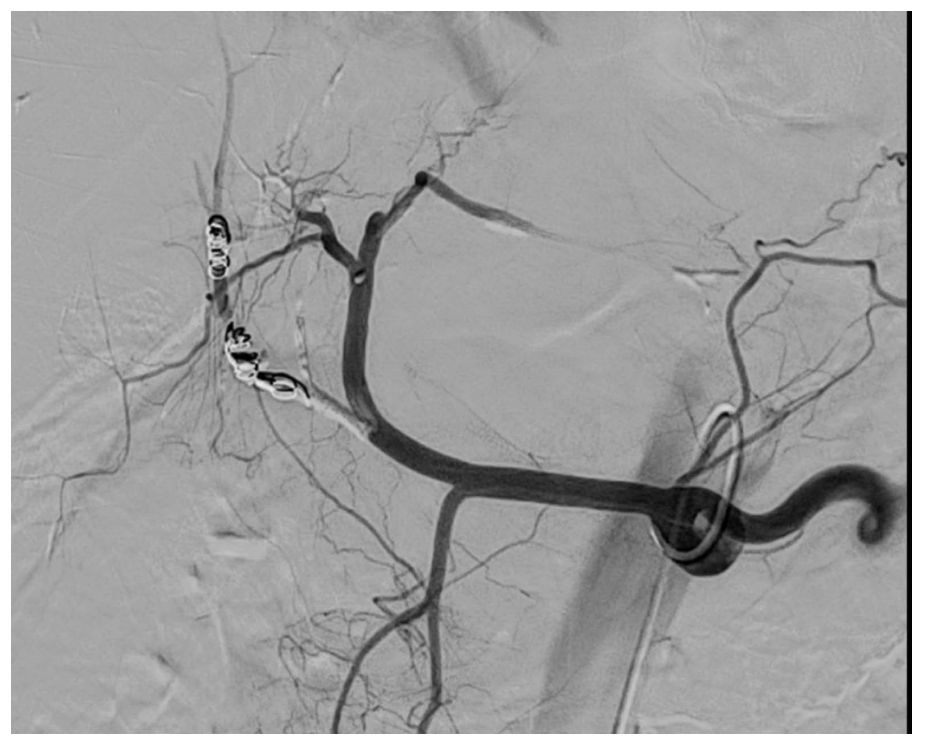

Figure 5. The right hepatic artery was embolized by multiple coils at both the proximal and the distal sides of the two pseudoaneurysms.

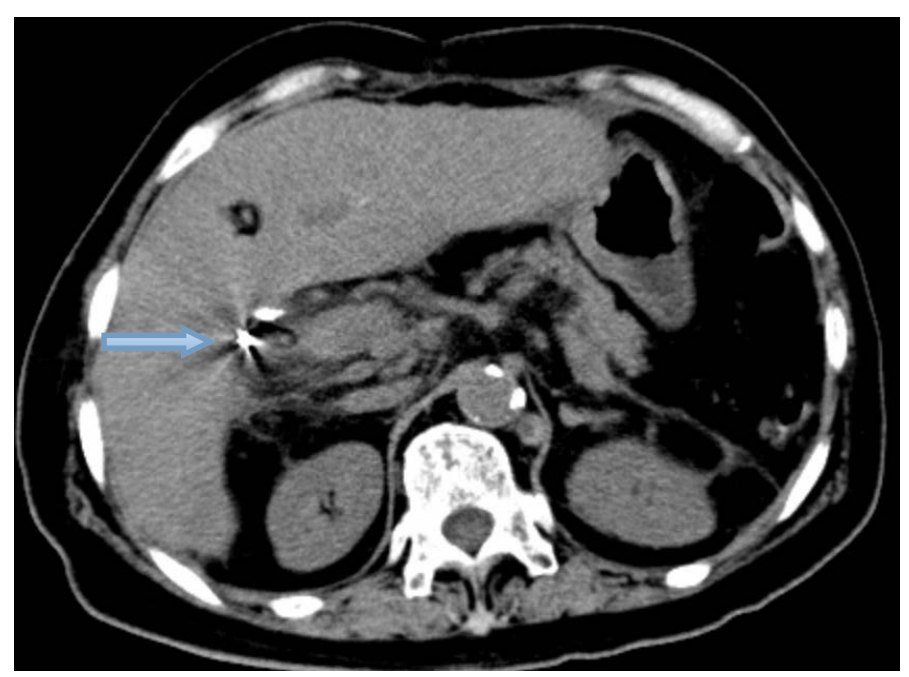

Figure 6. CT examination showed multiple coils at the psedoaneurysm sites one month after TAE (arrow). 


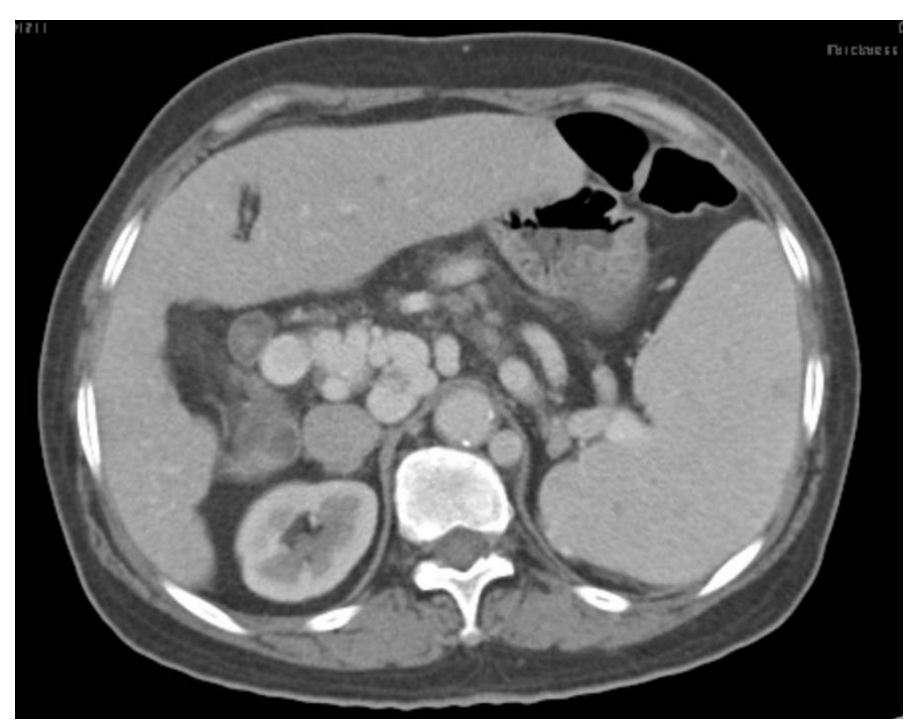

Figure 7. No pseudoaneurysm on the CT imagines before first biliary surgery two years ago.

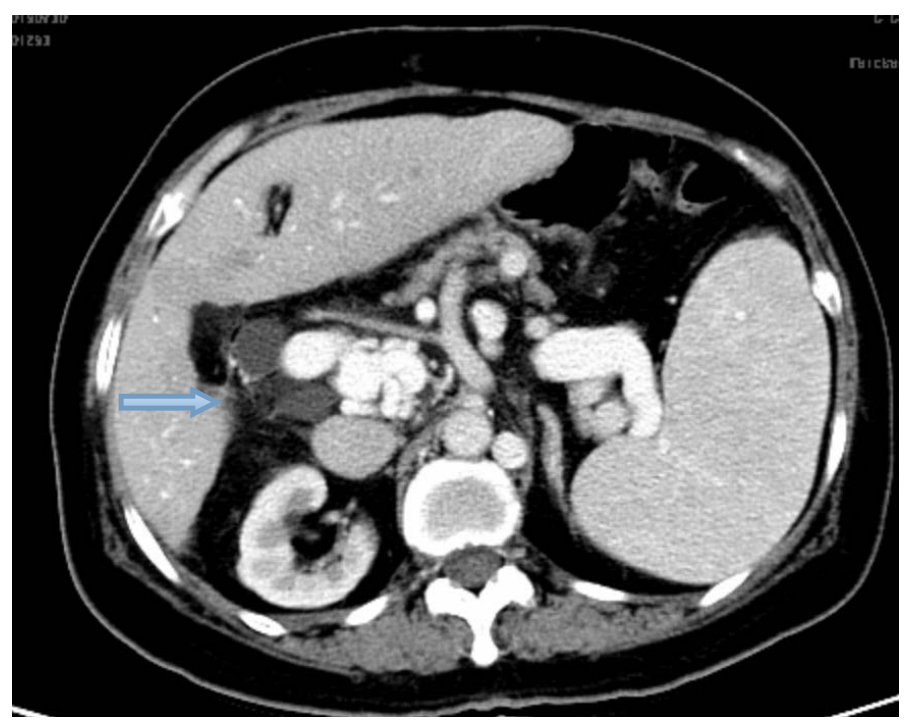

Figure 8. Two small pseudoaneurysms were visualized on the CT imagines two years after first biliary surgery (arrow).

We have reported three giant hepatic artery aneurysms leading to abdominal bleeding, which might be associated with arteriosclerosis [8]. In the present case we postulate that the two small hepatic pseudoaneurysms were most likely caused by the injuries from her first cholecystectomy and choledocholithotomy, since they were absent in the first surgery, but they were present two years later as shown in the CT images. This case provides the evidence that surgery is one of etiological causes for pseudoaneurysm formation.

While Quincke's triad of abdominal pain, jaundice and gastrointestinal bleeding is the typical presentation of hemobilia, the complete triad only accounts for less than $40 \%$ of patients [6]. Indeed, the hemobilia in our case manifested itself as sudden bleeding through the T-tube. It has been reported that direct observation of bleeding from the ampulla of Vater by endoscopy examination or endoscopic retrograde cholangiopancreatography can confirm the diagnosis of hemobilia [9]. However, in the present case, we could not find any active bleeding or any abnormality site in the whole biliary tract by choledochoscope examination, which suggests that endoscope examination is not the best choice as a confirmation method to find the cause of all hemobilia. In fact, selective celiac and superior mesenteric artery angiography has shown to be more reliable in many cases [10]. In agree- 
ment with this, selective celiac angiography clearly revealed the two small hepatic pseudoaneurysms in our case.

\section{Conclusion}

There are several options to treat hepatic pseudoaneurysm by surgery and/or interventional radiology. It has been reported that success rate for angiographic control of hemobilia by TAE is $80 \%-100 \%$ [11]. But, in some reports, radiologic interventions proved to be failed and surgical intervention was needed for the treatment of hemobilia [1] [12]. In our present case radiologic embolization has achieved a good result with no bleeding recurrence.

\section{References}

[1] Takeda, K., Tanaka, K., Endo, I., Togo, S. and Shimada, H. (2010) Two-Stage Treatment of an Unusual Haemobilia Caused by Intrahepatic Pseudoaneurysm. World Journal of Hepatology, 2, 52-54.

[2] Murugesan, S.D., Sathyanesan, J. and Lakshmanan, A. (2014) Massive Hemobilia: A Diagnostic and Therapeutic Challenge. World Journal of Surgery, 38, 1755-1762. http://dx.doi.org/10.1007/s00268-013-2435-5

[3] Petrou, A., Brennan, N., Soonawalla, Z. and Silva, M.A. (2012) Hemobilia Due to Cystic Artery Stump Pseudoaneurysm Following Laparoscopic Cholecystectomy: Case Presentation and Literature Review. International Surgery, 97, 140-144. http://dx.doi.org/10.9738/CC52.1

[4] Julianov, A. and Georgiev, Y. (2014) Hepatic Artery Aneurysm Causing Obstructive Jaundice. Quantitative Imaging in Medicine and Surgery, 4, 294-295.

[5] Murugesan, S.D., Sathyanesan, J., Lakshmanan, A., Ramaswami, S., Perumal, S. and Perumal, S.U. (2014) Massive Hemobilia: A Diagnostic and Therapeutic Challenge. World Journal of Surgery, 38, 1755-1762. http://dx.doi.org/10.1007/s00268-013-2435-5

[6] Petrou, A., Brennan, N., Soonawalla, Z. and Silva, M.A. (2012) Hemobilia Due to Cystic Artery Stump Pseudoaneurysm Following Laparoscopic Cholecystectomy: Case Presentation and Literature Review. International Surgery, 97, 140-144. http://dx.doi.org/10.9738/CC52.1

[7] Lee, Y.-T., Lin, H., Chen, K.-Y., Wu, H.S., Hwang, M.H. and Yan, S.L. (2010) Life-Threatening Hemobilia Caused by Hepatic Pseudoaneurysm after T-Tube Choledochostomy: Report of a Case. BMC Gastroenterology, $10,81$. http://dx.doi.org/10.1186/1471-230X-10-81

[8] Liu, Z.J., Yang, S.Y., Xi, P.C., Aiman, Shi, Z.L. and Bian, J.M. (2014) Surgical Treatment of Giant Aneurysm of Hepatic Artery: Report of Three Cases. Hepatogastroenterology, 61, 1192-1195.

[9] Hendriks, M.P., Wanten, G.J. and Drenth, J.P. (2009) Management of Hemobilia and Pancreatitis after Liver Biopsy: A Key Role for Endoscopic Retrograde Cholangiopancreaticography. Liver Transplantation, 15, 1653-1654. http://dx.doi.org/10.1002/lt.21807

[10] Panda, N., Narasimhan, M., Gunaraj, A. and Ardhanari, R. (2014) Laparoscopic Management of Post-Cholecystectomy Sectoral Artery Pseudoaneurysm. Journal of Minimal Access Surgery, 10, 37-39. http://dx.doi.org/10.4103/0972-9941.124471

[11] Sibulesky, L., Ridlen, M. and Pricolo, V.E. (2006) Hemobilia Due to Cystic Artery Pseudoaneurysm. American Journal of Surgery, 191, 797-798. http://dx.doi.org/10.1016/j.amjsurg.2005.07.041

[12] Paseka, T., Vlček, P., Vojtíšek, B. and Kianička, B. (2012) Right Hepatic Artery Pseudoaneurysm Fistulating to the Biliary Tract Responsible for Recurrent Upper Gastrointestinal Tract Bleeding. Cas Lek Cesk, 151, 472-475. 